\title{
A Functional Anatomy Based Kinematic Human Hand Model with Simple Size Adaptation
}

\author{
Frank P. J. van der Hulst, Simon Schätzle, Carsten Preusche and André Schiele
}

\begin{abstract}
For the purpose of ergonomic human-machine interaction and geometrical design of hand held haptic devices, a kinematic model that represents the functional anatomy of different human hands is desired.

It is the goal of this paper to present a kinematic hand model that is based on human physiology and that is easily adaptable to represent various real human hand sizes. This is achieved by exploiting body proportions to derive finger segment lengths from the hand length.

A partial hand model validation, involving index- and middle finger validation using a group of subjects, indicates that the use of body proportions offers a good estimate of finger length from a given hand length. Model estimated fingertip positions over a motion trajectory remain within reasonable limits when compared with experimental data for this subject group.

The model is promising for usage in practical situations since only hand length, which is easy to measure or to obtain from literature, is required as an input. Phalange lengths, which are sparsely available from literature and difficult to measure, are generated by the model.
\end{abstract}

\section{INTRODUCTION}

$\mathbf{M}$ ANY robotic devices are designed for interaction with humans and in particular for interaction with human hands. A typical example is the field of haptics for teleoperation and rehabilitation purposes, where interfaces and exoskeletons interact with, or connect to, the hand and fingers. Such devices are required to provide ergonomic human-machine interaction, not constraining natural movement and workspace during motion.

The complicated nature of the human hand raises the desire for a truly kinematic model of the hand, based on the physiology of its joints. Such a model could find its use in ergonomic human-machine interaction design for optimization of kinematic structures and geometrical design of hand held objects. Also, the hand model could be used to evaluate realistic hand functionality in the design of devices such as prosthetics and humanoid end-effectors. Another application of a hand model lies in estimating the state of a physical human hand. Forward kinematics can be applied to express the posture as function of the joint angles. The other way around, inverse kinematics can be used to derive joint angles from a given posture.

F. P. J. van der Hulst and A. Schiele are with the Telerobotics and Haptics Laboratory, European Space Research and Technology Centre, European Space Agency, 2201 AZ Noordwijk, The Netherlands, and with the Delft Biomechanical Engineering Department, Mechanical Engineering Faculty, Delft University of Technology, 2628 CD Delft, The Netherlands. Frank.van.der.Hulsteesa.int, Andre.Schieledesa.int

S. Schätzle and C. Preusche are with the Institute of Robotics and Mechatronics, German Aerospace Center (DLR). simon.schaetzleedlr.de
For the hand to be able to grasp and to hold objects, the ability of the thumb to oppose each single finger is essential. This functionality is termed thumb-finger opposition and is the result of multiple factors that will be discussed in Section II. Multiple published hand models will be summarized, yet none of these combines all factors required to achieve natural thumb-finger opposition.

In addition to the observed limitations on natural thumbfinger oppositions, there is another factor limiting the practical use of hand models. While in an experimental environment a calibrated model might be usable, in a practical application it is often required to vary its dimensions. One can think of many applications, such as for instance: optimizing human-machine interaction for different operators, evaluating ergonomic object interaction for different users or calculating forward or inverse kinematics for different subjects.

It is the goal of this paper to present a kinematic hand model that is based on the real functional anatomy of the human hand and that is easily adaptable to represent different physical hand sizes. The applied approach is to make use of body proportions for segment length estimation. A partial validation of the model will be performed for a scenario where the finger end-point position is estimated from a given set of joint angle measurements for various subjects.

This paper is organized as follows. Section II describes the functional anatomy of the human hand. Section III covers the construction and the parameterization of the kinematic model. Section IV describes validation of finger length estimation and optimization of model parameterization. Section $\mathrm{V}$ reports on model validation by finger end-point estimation. Section VI presents the conclusion and future work.

\section{ANATOMY OF THE HUMAN HAND}

For clarifying the terminology, Fig. 1 shows the anatomical position of the hand and the movement conventions.

\section{A. Bony Structure}

The human hand is composed of 27 bones, arranged in 5 serial kinematic chains forming the fingers. The fingers are numbered as follows. 1: thumb, 2: index finger, 3: middle finger, 4: ring finger and 5: little finger. Each finger (2-5) consists of a metacarpal bone located in the hand and 3 phalanges named the proximal-, medial, and distal phalange (in the order from finger base to fingertip). The thumb only consists of a metacarpal and 2 phalanges; it does not have a medial phalange. The remaining 8 hand bones are the carpals, located in the wrist. 

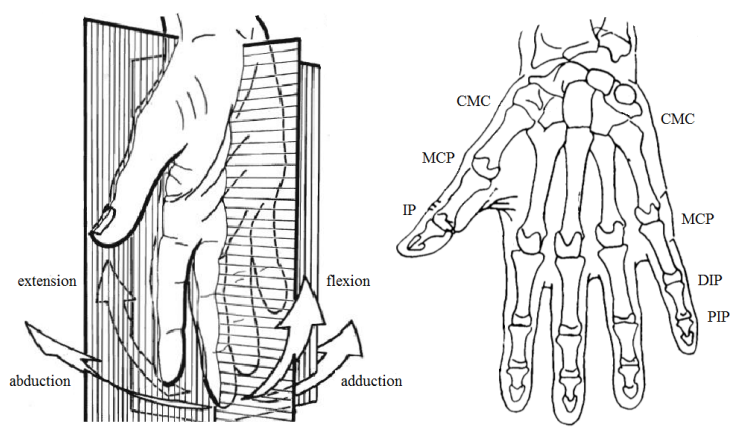

Fig. 1. Anatomical position of the hand. Left: pure flexion-extension takes place in the horizontally hatched sagittal plane. Pure adduction-abduction takes place in the vertically hatched frontal plane. Adapted from [1]. Right: bone structure of the hand with joint names indicated. Adapted from [3].

The names of the joints depend on the bones they link. In the right half of Fig. 1 the bone structure and the following joints can be seen: carpometacarpal joint (CMC), metacarpophalangeal joint (MCP) and interphalangeal joints (IP). In the fingers the two IP joints are distinguished by the prefixes, proximal (PIP) and distal (DIP).

\section{B. Hand Models - State of the Art}

In the human hand, the thumb is able to oppose each finger. This functionality is termed thumb-finger opposition and is essential to grasp and to hold objects. It is achieved by: the placement of the thumb anterior to (in front of) the palm and the fingers, automatic opposition resulting from oblique flexion of the fingers by inclined axes of rotation, and the hollowing of the palm [1]. The two latter effects will be described in Section II-C.3.

In the $24 \mathrm{DoF}$ model in [2] and the $23 \mathrm{DoF}$ model in [3] effects of palm hollowing have been implemented as CMC flexion-extension. While this allows arcing of the palm, the displacement is purely with respect to the frontal plane. No palm arcing effects have been included in the $26 \mathrm{DoF}$ model in [4] where only CMC ad-/abduction within the frontal plane was implemented, and in [5] where a 16 DoF model with a rigid palm is presented.

The natural opposition effects in the thumb were described in [1]. This was modeled using $5 \mathrm{DoF}$ in [3]. In all the other named hand models and the thumb model in [6], the thumb functionality is approximated by 4 DoFs.

While multiple of the mentioned models offer pure CMC flexion-extension or ad-abduction, none combines these motions for a more natural hollowing of the palm. In all models, finger flexion-extension takes place purely in the sagittal plane, and thus no oblique flexion is supported. Judging from this information, none of the models combines the factors that are essential to achieve natural thumb-finger opposition.

\section{Functional Anatomy}

As a result of the specific anatomy of the joints in the hand, objects can be grasped stably. Most of the information in this section has been adopted from [7], where hand and finger anatomy effects from [1] have been summarized.
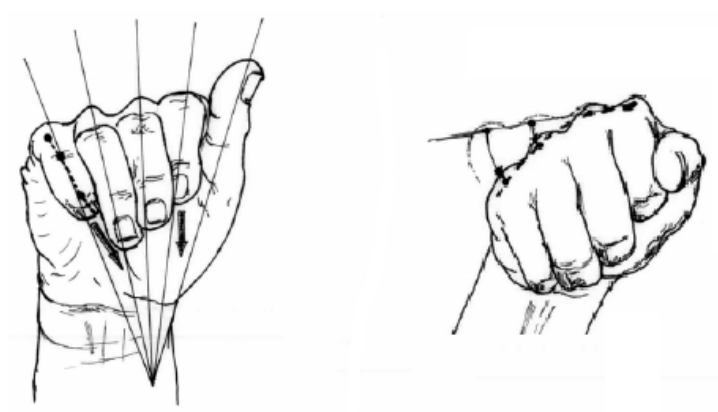

Fig. 2. Thumb-finger opposition effects. Left: automatic opposition by oblique finger flexion caused by inclined flexion-extension axes in the MCP, PIP and DIP joints. Right: hollowing of the palm by movement of the metacarpal heads in the CMC joints, with respect to the frontal plane (anteriorly) and slightly to the side (laterally).

1) Thumb Joints Anatomy: The thumb CMC is a saddle joint that offers 2 DoF: flexion-extension and antepositionretroposition (moving the thumb in front of the hand and moving it back). The axes of rotation are perpendicular and cross each other, yet do not intersect. Therefore no axial rotation is possible. The MCP is a condyloid (ellipsoidalsocket) joint that offers 2 DoF: flexion-extension and ad/abduction. A 3rd DoF is available by means of a slight pronation-supination (axial rotation). The IP is a $1 \mathrm{DoF}$ hinge joint offering flexion-extension with a slight pronation caused by the inclination of the axis.

2) Finger Joints Anatomy: The finger MCP joints are condyloid joints with 2 DoF: flexion-extension with respect to the frontal plane and ad-/abduction with respect to the sagittal plane. The available axial rotation is only passive. The IP joints are the PIP and DIP, both offering $1 \mathrm{DoF}$ flexion-extension. Depending on the finger, the flexionextension axes are inclined, introducing motions directed sideways (lateral) and axial rotation [8].

3) Thumb-finger Opposition: The anatomical effects in the joints combine into the following two mechanisms that contribute to thumb-finger opposition.

Automatic opposition: During flexion, the fingers are directed towards the same point (the radial pulse) as shown in the left half of Fig. 2. This effect presents the pulp of the fingers to that of the thumb and to the object to grasp. The result is an increased contact surface contributing to the strengthening of the grip.

Automatic opposition results from inward finger flexion caused by the MCP flexion axis inclination and oblique finger segment flexion caused by the PIP and DIP flexion axes inclination. The PIP and DIP flexion axes are perpendicular to the long axis of the bone in full extension and become progressively more oblique during flexion [8]. This is an effect of asymmetry of the bone surfaces moving with respect to each other in the joints (the articular surfaces) and of the different tensions in the ligaments. Oblique flexion causes the finger segments not to flex in the sagittal plane, yet in an increasingly oblique plane.

The effects of inward finger flexion and oblique finger segment flexion increase from the index finger to the little 
finger, as also shown in the left half of Fig. 2.

The hollowing of the palm: The heads of the metacarpal bones located in the CMC joints move with respect to the frontal plane (anteriorly) and slightly to the side (laterally). As illustrated in the right half of Fig. 2, this effect increases from the index finger (where it is negligible) to the little finger, causing hollowing of the palm.

\section{HUMAN HAND MODEL}

Using the functional anatomy of the hand, described in the previous section, a kinematic model description is defined.

\section{A. Kinematic Structure}

The kinematic structure of the hand model is defined by base transformations relating the finger bases to the hand base and by Denavit-Hartenberg (DH) parameters describing the kinematic chain of each finger. This model includes the following human hand joints.

Index- and middle finger: Both consist of 4 DoF. 2 DoF MCP: flexion-extension $\left(\theta_{1}\right)$ and ad-abduction $\left(\theta_{2}\right), 1$ DoF PIP: flexion-extension $\left(\theta_{3}\right)$, and 1 DoF DIP: flexionextension $\left(\theta_{4}\right)$.

Ring- and little finger: Both consist of 5 DoF. 1 DoF CMC: lateral (sideways) flexion-extension $\left(\theta_{1}\right), 2$ DoF MCP: flexion-extension $\left(\theta_{2}\right)$ and ad-abduction $\left(\theta_{3}\right), 1$ DoF PIP: flexion-extension $\left(\theta_{4}\right)$, and 1 DoF DIP: flexion-extension $\left(\theta_{5}\right)$.

Thumb: The thumb model consists of $6 \mathrm{DoF}$, enabling realistic thumb-finger opposition. 2 DoF CMC: flexion-extension $\left(\theta_{1}\right)$ and ad-abduction $\left(\theta_{2}\right)$ (antepositionretroposition). These axes cross but do not coincide, forming a saddle joint. 3 DoF MCP: flexion-extension parallel to the previous flexion axis $\left(\theta_{3}\right)$, abduction-adduction parallel to the previous abduction axis $\left(\theta_{4}\right)$, and axial rotation collinear with the proximal finger segment $\left(\theta_{5}\right)$. 1 DoF IP: flexionextension $\left(\theta_{6}\right)$.

The kinematic structure is shown in Fig. 3. The finger base frames $C M C_{1}, M C P_{2,3}$, and $C M C_{4,5}$ (with the indices 1-5 indicating the fingers) are expressed with respect to the hand base frame $O_{b}$ via the transformations $T_{C M C 1}^{b}$, $T_{M C P 2,3}^{b}$, and $T_{C M C 4,5}^{b}$ respectively. Each transformation is composed of a translation and a rotation. The rotation matrices are given in (1) for the thumb, in (2) for the indexand middle finger, and in (3) for the ring- and little finger. The notation convention and rotation matrices: $R_{x}, R_{y}$, and $R_{z}$, are according to [9].

$$
\begin{gathered}
R_{C M C 1}^{b}=R_{x}\left(\beta_{t}\right) R_{z}\left(-\alpha_{f}\right) R_{y}\left(-\alpha_{e}\right) \\
R_{M C P 2,3}^{b}=R_{y}\left(\phi_{m c p}\right) \\
R_{C M C 4,5}^{b}=R_{y}\left(\phi_{m c p}\right) R_{x}\left(\beta_{h}\right)
\end{gathered}
$$

The transformations from the fingertip to the finger base include the variable joint angles $\theta_{i}$ and are described using the DH-parameters given in Table I for each finger.

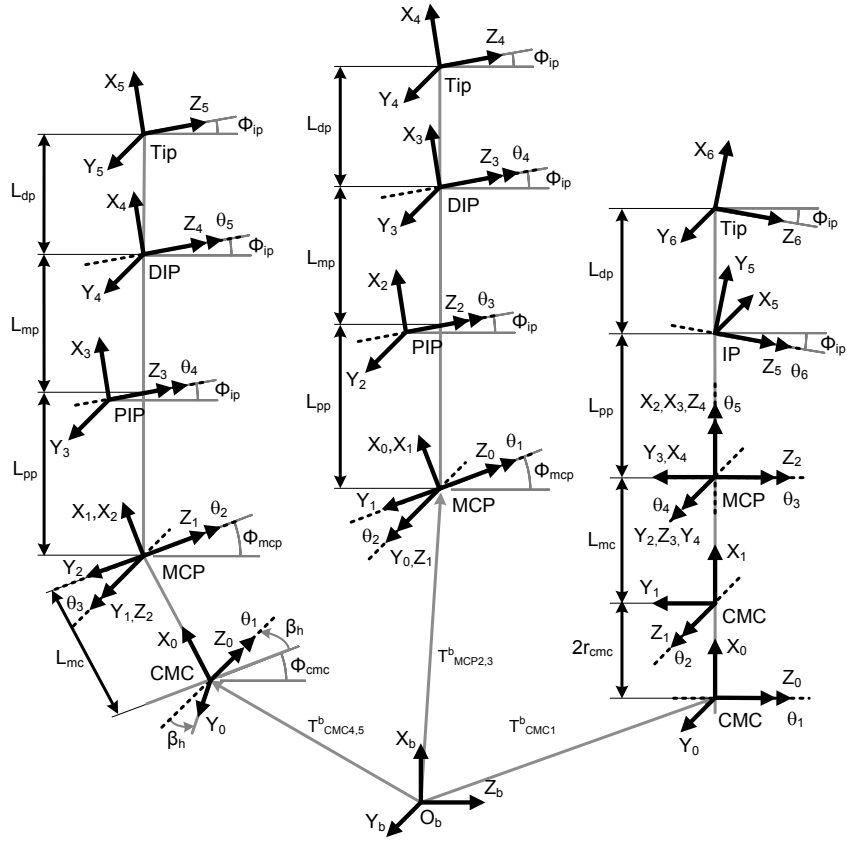

Fig. 3. Hand model kinematic structure frontal view. For visual clarity, thumb base rotations (1), and finger chain parameter indices are not shown.

TABLE I

DENAVIT-HARTENBERG PARAMETERS

\begin{tabular}{ccccc}
\multicolumn{5}{c}{ Thumb } \\
\hline Joint & $a_{i}[\mathrm{~m}]$ & $d_{i}[\mathrm{~m}]$ & $\alpha_{i}\left[^{\circ}\right]$ & $\theta_{i}\left[^{\circ}\right]$ \\
\hline$T_{1}^{0}$ & $2 r_{c m c}$ & 0 & -90 & $\theta_{1}$ \\
$T_{2}^{1}$ & $L_{m c^{-}-r_{c m c}}$ & 0 & 90 & $\theta_{2}$ \\
$T_{3}^{2}$ & 0 & 0 & -90 & $\theta_{3}$ \\
$T_{4}^{3}$ & 0 & 0 & 90 & $\theta_{4}+90$ \\
$T_{5}^{4}$ & 0 & $L_{p p}$ & $90-\phi_{i p}$ & $\theta_{5}-90$ \\
$T_{6}^{5}$ & $L_{d p} C_{i p}$ & $L_{d p} S_{i p}$ & 0 & $\theta_{6}+90$ \\
\hline
\end{tabular}

Index and Middle Finger

\begin{tabular}{ccccc}
\hline Joint & $a_{i}[\mathrm{~m}]$ & $d_{i}[m]$ & $\alpha_{i}\left[^{\circ}\right]$ & $\theta_{i}\left[^{\circ}\right]$ \\
\hline$T_{1}^{0}$ & 0 & 0 & -90 & $\theta_{1}$ \\
$T_{2}^{1}$ & $L_{p p} C_{i p}$ & 0 & 90 & $\theta_{2}+\phi_{i p}-\phi_{m c p}$ \\
$T_{3}^{2}$ & $L_{m p} C_{i p}$ & $\left(L_{p p}+L_{m p}\right) S_{i p}$ & 0 & $\theta_{3}$ \\
$T_{4}^{3}$ & $L_{d p} C_{i p}$ & $L_{d p} S_{i p}$ & 0 & $\theta_{4}$ \\
\hline
\end{tabular}

\begin{tabular}{ccccc}
\multicolumn{5}{c}{ Ring and Little Finger } \\
\hline Joint & $a_{i}[\mathrm{~m}]$ & $d_{i}[m]$ & $\alpha_{i}\left[^{\circ}\right]$ & $\theta_{i}\left[^{\circ}\right]$ \\
\hline$T_{1}^{0}$ & $L_{m c}$ & 0 & $-\beta_{h}$ & $\theta_{1}$ \\
$T_{2}^{1}$ & 0 & 0 & -90 & $\theta_{2}$ \\
$T_{3}^{2}$ & $L_{p p} C_{i p}$ & 0 & 90 & $\theta_{3}+\phi_{i p}-\phi_{m c p}$ \\
$T_{4}^{3}$ & $L_{m p} C_{i p}$ & $\left(L_{p p}+L_{m p}\right) S_{i p}$ & 0 & $\theta_{4}$ \\
$T_{5}^{4}$ & $L_{d p} C_{i p}$ & $L_{d p} S_{i p}$ & 0 & $\theta_{5}$ \\
\hline
\end{tabular}

Joint angles: $\theta_{1}, \theta_{2}, \theta_{3}, \theta_{4}, \theta_{5}, \theta_{6}$; Phalange lengths: $L_{m c}, L_{p p}, L_{m p}$, $L_{d p}$; Axis inclination angles: $\phi_{i p}, \phi_{m c p}$; Thumb CMC radius: $r_{c m c}$; Ring- and little finger base rotation offset: $\beta_{h}$; Shorthand notations: $S_{i p}=$ $\sin \left(\phi_{i p}\right), C_{i p}=\cos \left(\phi_{i p}\right)$. DH-convention according to [9]. 
The thumb CMC saddle joint axes are placed at a distance $r_{c m c}$ at either side of the joint center. This accounts for the joint head diameter.

The anterior placement of the thumb is defined by thumb base frame rotation of $\beta_{t}$, inclination of the thumb in its frontal plane $\alpha_{f}$ and inclination of the thumb in its sagittal plane $\alpha_{s}$. These rotations are described in (1), where $\alpha_{e}$ is the thumb elevation, given by:

$$
\alpha_{e}=\tan ^{-1}\left(\tan \left(\alpha_{s}\right) \cos \left(\alpha_{f}\right)\right) .
$$

Palm hollowing is implemented for the ring- and little finger via a finger base rotation offset $\beta_{h}$ and via the flexionextension axes inclination angles $\phi_{c m c}$ in the CMC joints. Since the CMC joint motion is negligible for the index- and middle finger, their CMC joints are not modeled.

Automatic finger-thumb opposition by inward finger flexion and oblique finger segment flexion are implemented via the flexion-extension axes inclination angles $\phi_{m c p}$ and $\phi_{i p}$ respectively.

\section{B. Model Parameters}

In this section, the parameter values are defined. Distinction is made between parameters that are derived from body proportions, that are assumed a value, and that are unknown. Although the latter two categories contain rough estimates and unknown parameter values, the model is implemented such that it is ready to accept new parameter values whenever these come available.

1) Parameters Derived from Body Proportions: Simple adaptation of the model to represent different hand sizes is achieved by generating the link lengths $L_{p p}, L_{m p}$, and $L_{d p}$ from a given hand length. A similar approach was used in [3], where dimensional measurements, expressed as percentage of hand length, breadth and thickness, were used to scale a hand model for different hand sizes. This approach is based on the assumption that normal hands maintain anatomical structure and dimensional proportions, regardless of their physical size [8].

The advantage is that the hand length, which is easy to measure or to obtain from literature, is used to generate link lengths that are difficult to measure and sparsely reported.

Table II presents all finger phalange lengths as percentages of the hand length. This conversion table was reported in [10] and is based on 32 subjects (15 male, 17 female). In Section IV this table will be optimized using experimental data.

TABLE II

Hand Length to Phalange Length Conversion Table [10]

\begin{tabular}{lccc}
\hline & Proximal $\left(L_{p p}\right)$ & Medial $\left(L_{m p}\right)$ & Distal $\left(L_{d p}\right)$ \\
\hline Thumb & 17.1 & - & 12.1 \\
Index finger & 21.8 & 14.1 & 8.6 \\
Middle finger & 24.5 & 15.8 & 9.8 \\
Ring finger & 22.2 & 15.3 & 9.7 \\
Little finger & 17.7 & 10.8 & 8.6 \\
\hline
\end{tabular}

Each entry represents phalange length as percentage of hand length.
2) Assumed Parameters: Since no detailed quantitative information has been found, the axis inclination angles $\left(\phi_{c m c}, \phi_{m c p}, \phi_{i p}\right)$, the thumb parameters $\left(\beta_{t}, \alpha_{f}, \alpha_{s}, r_{c m c}\right)$, and the palm hollowing base orientation $\beta_{h}$ are assumed based on qualitative knowledge of the anatomy of the hand described in [1]. The values used in the hand model are as proposed in [7].

The IP axis inclination angle $\phi_{i p}$ is approximately $5-10^{\circ}$ for the thumb [1], therefore a value of $7.5^{\circ}$ is assumed in the model. The same source reports that the automatic opposition by inward and oblique flexion increases from the index finger, where it is negligible, to the little finger. Therefore the finger IP inclination angles are assumed increasing from the index- to the little finger: $0^{\circ}, 2^{\circ}, 4^{\circ}, 8^{\circ}$. Thus ranging up to approximately the value for the thumb.

The MCP axes inclination angles $\phi_{m c p}$ are assumed such that all fingers are directed towards the radial pulse when flexed (left half of Fig. 2). The MCP axis inclination angles are assumed: $-6.8^{\circ}, 3.6^{\circ}, 13.8^{\circ}, 23.9^{\circ}$ for the index- to the little finger, as proposed in [7]. The CMC axes inclination angles $\phi_{c m c}$ of the ring- and little finger are assumed equal to their $\phi_{m c p}$ angles.

The thumb base rotation offset $\beta_{t}$ is assumed $-90^{\circ}$ and the projection angles $\alpha_{f}$ and $\alpha_{s}$ are assumed $30^{\circ}$ and $40^{\circ}$ respectively in the neutral thumb position [1]. The $\mathrm{CMC}$ saddle joint head radius $r_{c m c}$ is estimated to be $5 \mathrm{~mm}$.

The finger base rotation offset for palm hollowing $\beta_{h}$ is set to $45^{\circ}$.

3) Unknown Parameters: No reliable quantitative information defining all finger base positions with respect to the hand base has been found. Therefore no finger base origin translations are proposed at this moment.

Also the metacarpal segment length $L_{m c}$ is not assigned a value currently, it could be derived from body proportions in the future, analogue to the phalangeal segment lengths in Section III-B.1.

\section{EXPERIMENTAL OPTIMIZATION OF THE HAND MODEL}

As described in Section III-B.1, Table II can be used to calculate all phalange lengths from a given hand length. This table, which was obtained from literature, was validated and optimized using experimental data. As a result, an updated conversion table for the index- and middle finger is presented, which will be applied in Section $\mathrm{V}$ during a partial model validation.

\section{A. Method}

For both the index- and middle finger, the terms in the original conversion table were multiplied by a correction factor, ensuring that the length relation between the phalanges holds, while the sum of phalange lengths now matches the finger length estimated from experimental data.

This method required the finger length to be estimated from experimental data, using a motion tracking experiment to identify the fingertip and base positions. 


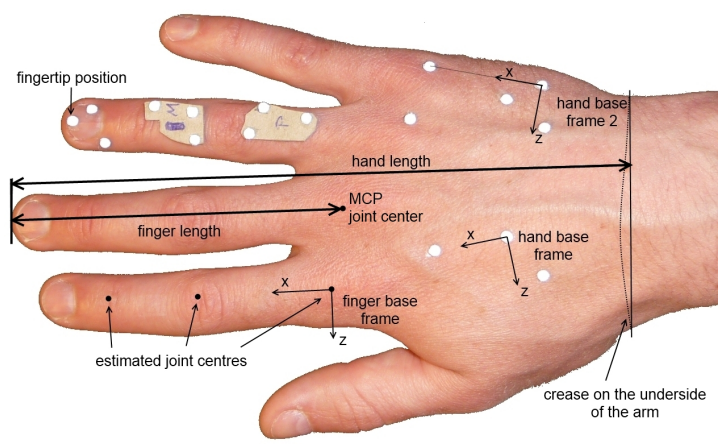

Fig. 4. Attachment of markers on one finger and the hand.

1) Experimental Setup: An optical motion capture system with passive markers was used for the tracking of the subjects' finger movements. Seven Vicon ${ }^{\circledR}$ MX3+ cameras were installed such that the reflective markers on the back of the hand and on the fingers, placed as shown in Fig. 4, were tracked for the full range of finger motion. In order to minimize the effect of skin movement, markers were placed onto the finger segments rather than on the joints. These $3 \mathrm{~mm}$ passive markers had no observable effect on the natural finger motion. Application of two frames on the back of the hand allowed to track the hollowing of the hand.

2) Experimental Data: Cartesian marker positions were registered during index- and middle finger motion. From this data, joint center positions were estimated. Subsequently, the finger length was calculated as the norm of the vector from the estimated MCP joint center to the measured fingertip marker position on the fully stretched finger.

Datasets of two female and five male subjects aged from 26 to 30 years were collected. Table III lists the subject hand parameters, including the hand length, measured as shown in Fig. 4.

After markers had been attached to the hand, a predefined trajectory composed of three phases was executed by the subjects with each finger subsequently. After the initial posture in which the fingers were fully stretched, the motion trajectory started with a flexion-extension, followed by an ad-/abduction with the finger stretched, and ended with a circumduction of the stretched finger, performing both flexionextension and ad-/abduction of the MCP joint. This trajectory involved all relevant finger degrees of freedom. During this routine, marker positions were tracked and stored.

\section{B. Data Processing}

In the first part of the data processing, joint center positions were estimated. Based on this result, joint angles sets for each finger configuration will be calculated in Section V.

TABLE III

SubJeCt HAND DimENSIONS

\begin{tabular}{lccccccc}
\hline Subject & 1 & 2 & 3 & 4 & 5 & 6 & 7 \\
\hline Hand length $[\mathrm{mm}]$ & 170 & 177 & 189 & 200 & 192 & 200 & 198 \\
Hand breadth $[\mathrm{mm}]$ & 69 & 75 & 85 & 82 & 85 & 89 & 82 \\
\hline
\end{tabular}

1) Estimation of Joint Centers: Joint center positions were derived from measured data by analyzing the movement of the adjoining finger segments. Markers were rotated from their initial position, around a specific inclination axis, until coinciding with the markers on the flexed segment.

The axes inclinations defined in the hand model were used for this estimation of joint center positions. In order to assure that the joint angles extracted from the measured data are comparable with the modeled data, the definition of joint axes inclinations should match between modeled and measured data. If this is not the case, a single fingertip position is described by different joint angle sets in measured and modeled data. For that reason, axes inclinations from the model were used for the following processing.

Joint center positions have been estimated by solving the following nested optimization problem: The cost function of the outer algorithm, namely the joint center position optimization, is defined as follows:

$$
\min _{\vec{x} \in \mathbb{R}^{3}} f(\vec{x}) \quad \text { with } f(\vec{x})=\sqrt{\text { sum }_{\text {posError }} / N}
$$

where $\vec{x}$ is the position vector of the estimated joint center, $N$ is the number of different measured finger flexions and sum $_{\text {posError }}$ is the result of the inner optimization algorithm. This inner optimization algorithm calculates a joint angle for each measured finger flexion with a given joint center from the outer optimization algorithm, so that the error between estimated and measured marker position is minimized as follows:

$$
\min _{q \in \mathbb{R}} f(q) \quad \text { with } f(q)=\underbrace{\sum_{m=1}^{M}\left\|P_{m, \text { meas }}-P_{m, \text { mod }}\right\|^{2} / M}_{\text {sumposError }}
$$

with $M$ the number of markers on the flexed finger segment, $P$ the Cartesian position vector from joint center to measured and estimated marker positions and $q$ the joint angle.

Within a loop, a joint angle is optimized for each finger flexion angle, and the squared errors are summed, resulting in sum $_{\text {posError }}$. This sum is the root mean square distance error between measured and estimated marker positions resulting from a rotation around an axis with a specific position and inclination.

2) Conversion Table Update: The finger length estimated from measurement, as described in Section IV-A.2 is termed reference finger length. The ratio between this length and the finger lengths from the conversion table (sum of phalange lengths), was taken as a correction factor for each subject and each finger. The table entries for each finger were multiplied by the corresponding correction factors so that an updated table resulted where the sum of phalange lengths is equal to the reference finger length.

In order to obtain one table that is applicable to the whole subject group, the conversion factors were averaged over all subjects for each finger. The updated conversion table is shown for the index- and middle finger in Table IV. 


\section{Discussion and Results}

Fig. 5 shows the errors in finger lengths calculated using the two conversion tables, with respect to the reference finger lengths from measurement.

Using the original conversion table, the finger length error is $93.3 \% \pm 3.8 \%$ and $94.6 \% \pm 4.1 \%$ (mean \pm standard deviation (s.d.)) for the index- and middle finger respectively.

The mean error shows that the generated finger lengths consistently have an offset from the reference finger length. This suggests that the original conversion table is not optimal for this subject group. The optimized conversion table corrects the mean offset, while the spread is kept equal. The low s.d. indicates that a constant conversion table is suitable to estimate finger lengths for different subjects. This shows that the approach of using body proportions can be exploited to estimate finger length from a given hand length.

The optimized conversion table is based on few subjects only. If tests show that this table does not hold for new subjects, it should be optimized for a larger subject group.

In the following section, prediction of finger end-point position will be tested, using the optimized conversion table as a new model baseline for phalange length parametrization.

\section{EXPERIMENTAL VALIDATION OF THE HAND MODEL}

An experimental partial validation of the hand model, consisting of the index- and middle finger, was conducted in order to validate its performance of finger end-point position estimation. Furthermore, this validation should indicate the feasibility of using body proportions to parameterize the phalange lengths using only the hand length as an input.

\section{A. Method}

The hand model takes joint angles and the hand length of each subject as an input and returns the modeled Cartesian end-point position as an output. In order to verify this predicted position, it was compared to the measured fingertip marker position. This was done for each subject over the motion trajectory described in Section IV-A.2.

For each point in time the end-point positions were measured. The corresponding sets of four joint angles for reaching this position were extracted from the measured marker positions by optimization and were then used as an input into the hand model for calculation of the corresponding model fingertip positions over time.

The same hardware setup and measurement data as described in Section IV has been used for this validation test.

TABLE IV

Updated Hand Length to Phalange Length Conversion Table

\begin{tabular}{lccc}
\hline & Proximal $\left(L_{p p}\right)$ & Medial $\left(L_{m p}\right)$ & Distal $\left(L_{d p}\right)$ \\
\hline Index finger & 23.5 & 15.2 & 9.3 \\
Middle finger & 26.0 & 16.8 & 10.4 \\
\hline Each entry represents phalange length as percentage of hand length.
\end{tabular}

Each entry represents phalange length as percentage of hand length.

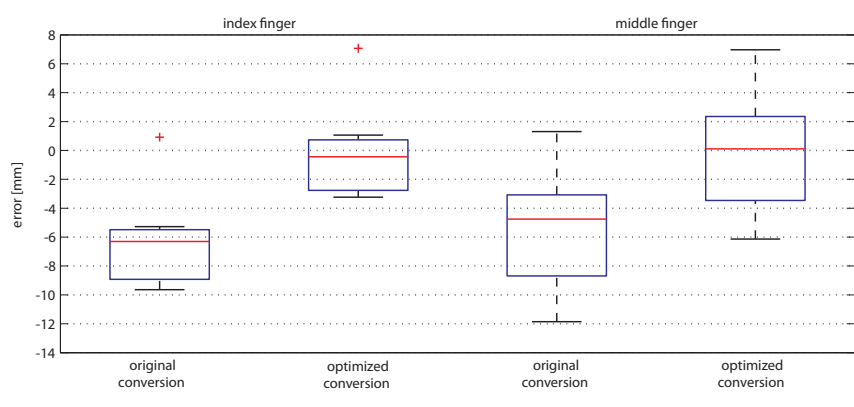

Fig. 5. Error of the finger lengths given by the sum of phalange lengths from the conversion tables, with respect to the reference finger length from measurements. Results are shown for both the original and updated conversion tables. All subjects $(\mathrm{N}=7)$.

\section{B. Data Processing}

Along with the joint centers from Section IV-B.1, joint angle sets based on the model defined axes inclinations have been calculated for each measured finger configuration.

In contrast to the estimation of joint center positions which uses only the markers of the adjoining finger segment, the optimization algorithm for calculating joint angle sets takes into account all finger markers, namely the full kinematic chain of one finger. The applied optimization algorithms are based on the methods described in [11] and [12].

For the comparison of measured and modeled data, a common base frame was required in order to represent marker positions. Each finger was assigned a finger base frame defined according to the hand model conventions and with an identical orientation for all fingers. Fingertip coordinates were then transformed and represented in the corresponding finger base frames. As shown in Fig. 4 the $\mathrm{x}$-axis was directed distal along the finger, the $\mathrm{y}$-axis was dorsal, and the $\mathrm{z}$-axis was in the frontal plane such that it completed a right-handed coordinate frame.

\section{Discussion and Results}

Fig. 6 shows the plots of $\mathrm{x}, \mathrm{y}$ and $\mathrm{z}$ components of the modeled and measured end-point positions during index finger motion (see Fig. 4 for axes definition). The corresponding error is shown in Fig. 7. This data is a typical result for one subject from the same subject group as used in Section IV.

The largest error occurs in the first third of the trajectory, which consists of a finger movement with intensive flexion. Due to the serial kinematics of the finger, small differences in finger segment lengths produce larger Cartesian end-point errors when the finger is flexed than when it is stretched. Furthermore it can be seen that the major error component is in the $\mathrm{z}$-direction. This indicates a possible mismatch between joint axes inclination assumptions of the model and real axis inclinations of the human hands.

Fig. 8 shows the results for all subjects combined. The mean absolute error of the Cartesian end-point estimation using the optimized conversion is $7.0 \pm 2.6 \mathrm{~mm}$ and 9.8 $\pm 2.5 \mathrm{~mm}$ for the index- and middle finger respectively. These results are valid for this subject group only, yet it can be seen that also the use of the original conversion from 

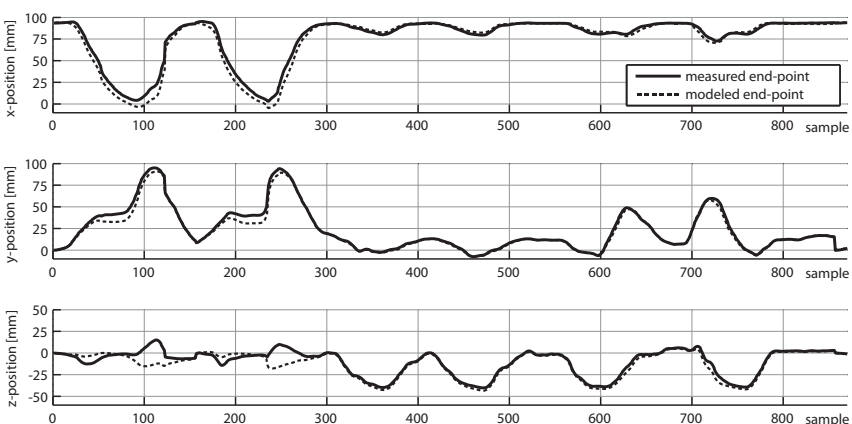

Fig. 6. Comparison of modeled and measured Cartesian fingertip position. Dataset from a single subject.
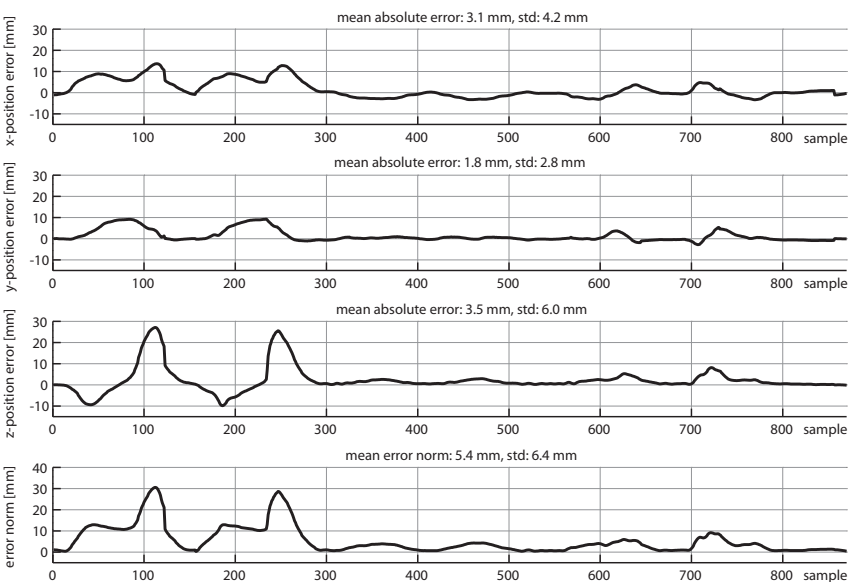

Fig. 7. Error of Cartesian fingertip position obtained from the model, with respect to that from measurement. Dataset from a single subject.

literature results in reasonable small errors, indicating that the approach of using body proportions is successful.

For the middle finger, the error is larger than for the index finger. This could suggest a larger mismatch in axis inclination angles in the middle finger.

\section{CONCLUSION AND FUTURE WORK}

A kinematic hand model based on the functional anatomy of the human hand was introduced. By the use of body proportions, the model is simple to adapt to different hand sizes, requiring only hand length as an input. The hand model returns an estimate of finger length and end-point position as was shown for the index- and middle finger.

Results suggest that body proportions can be exploited to derive phalange lengths from hand lengths. This approach was optimized for a subject group, showing improved results in finger length and end-point position estimation. The conversion table should be optimized for a larger subject group if the presented optimized conversion table does not hold for new subjects.

The error on end-point position estimation was found to be $7.0 \pm 2.6 \mathrm{~mm}$ and $9.8 \pm 2.5 \mathrm{~mm}$ for the index- and middle finger respectively. Validation results show that the mismatch between real and modeled axes inclinations forms the major contribution to this error. Further investigating the joint axes inclinations offers potential for improvement.

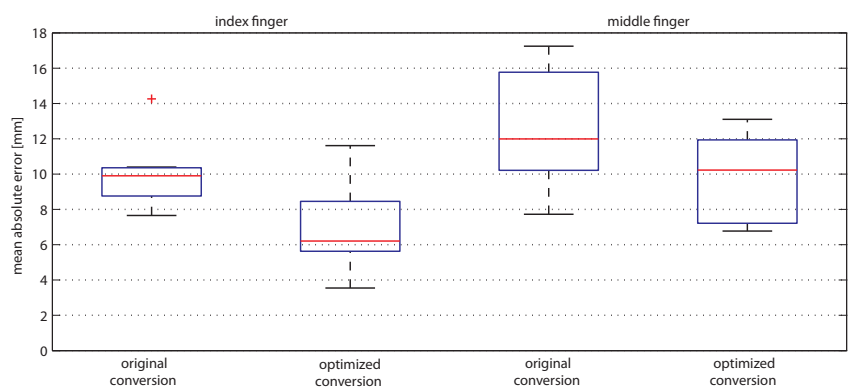

Fig. 8. Mean absolute error of Cartesian fingertip positions obtained from the model, with respect to those from measurements. All subjects $(\mathrm{N}=7)$.

Since the applied conversion was optimized for the same subject group as in the validation, follow-up experiments must show if similar model predictions can be achieved for new subjects. In future work, the whole hand model and its parameterization via body proportions should be validated. Including adaptation in hand width and palm hollowing.

The obtained results underline the practical use of the model by simple and quick adaptation to real human hand sizes, only requiring hand length as an input.

\section{ACKNOWLEDGMENTS}

The authors thank G. Stillfried from DLR for his support in the data processing and G. Gil Gómez for his illustrations (Fig. 2), summary of functional hand anatomy and the kinematic description derived from this.

\section{REFERENCES}

[1] I. Kapandji, The Physiology of the Joints, Churchill Livingstone, 1982.

[2] S. Cobos, M. Ferre, M. A. Sanchez Uran, J. Ortego and C. Pena, "Efficient human hand kinematics for manipulation tasks", in Proc. IEEE/RSJ International Conference on Intelligent Robots and Systems, 2008.

[3] N. Davidoff and A. Freivalds, "A graphic model of the human hand using Catia", in International Journal of Industrial Ergonomics, vol. 12, no. 4, pp. 255 - 264, 1993.

[4] H. Du and E. Charbon, "3D Hand Model Fitting for Virtual Keyboard System", in Proc. IEEE Workshop Applications of Computer Vision WACV, 2007.

[5] H. Hashimoto, H. Murakoshi, A. Sasaki, Y. Ohyama, K. Makino and S. Yokota, "Dynamical analysis of grasping with hand model for high quality product design", in Proc. SICE Annual Conf. 2010.

[6] K. Kim, Y. Youm, and W. K. Chung, "Human kinematic factor for haptic manipulation: the wrist to thumb," in Proc. of 10th Symp. Haptic Interfaces for Virtual Environment and Teleoperator Systems HAPTICS, 2002.

[7] G. Gil Gómez and A. Schiele, Compendium of Human Factors for Designing an Ergonomic Haptic Exoskeleton for the Human Hand, ESA internal documentation, 2006.

[8] E. Y. S. Chao, K-N. An, W. P. Cooney III and R. L. Linscheid, "Biomechanics of the hand. A basic research study." World Scientific. 1989

[9] B. Siciliano, L. Sciavicco, L. Villani, and G. Oriolo, Robotics Modelling, Planning and Control, Springer-Verlag Londen Limited, 2009.

[10] A. Freivalds, Biomechanics of the Upper Limbs: Mechanics, Modeling, and Musculoskeletal Injuries, Boca Raton; London: CRC Press, 2004.

[11] G. Stillfried, U. Hillenbrand, M. Settles and P. van der Smagt, "MRIbased skeletal hand movement model". The Human Hand - A Source of Inspiration for Robotic Hands, R. Balasubramanian and V. Santos (editors), Springer Tracts on Advanced Robotics, to be published.

[12] G. Stillfried and P. van der Smagt, "Movement model of a human hand based on magnetic resonance imaging (MRI)", in 1st International Conference on Applied Bionics and Biomechanics (ICABB), 14-16 Oct 2010, Venice, Italy. 